\title{
On the Herbrand content of LK
}

\author{
Bahareh Afshari Stefan Hetzl Graham E. Leigh \\ TU Wien, Austria \\ \{bahareh.afshari, stefan.hetzl, graham.leigh\}@tuwien.ac.at
}

\begin{abstract}
We present a structural representation of the Herbrand content of LK-proofs with cuts of complexity prenex $\Pi_{2} / \Sigma_{2}$. The representation takes the form of a typed non-deterministic tree grammar $\mathscr{G}$ of order 2 which generates a finite language, $L(\mathscr{G})$, of first-order terms that appear in the Herbrand expansions obtained through cut-elimination. In particular, for every Gentzen-style reduction $\pi \rightsquigarrow \pi^{\prime}$ between LK-proofs we study the induced grammars, respectively $\mathscr{G}$ and $\mathscr{G}^{\prime}$, and classify the cases in which language equality, $L(\mathscr{G})=L\left(\mathscr{G}^{\prime}\right)$, and language inclusion, $L(\mathscr{G}) \supseteq L\left(\mathscr{G}^{\prime}\right)$, hold.
\end{abstract}

\section{Introduction}

In classical first-order logic a proof can be considered as being composed of two layers: on the one hand the terms by which quantifiers are instantiated, and on the other hand, the propositional structure. This separation is most clearly illustrated by Herbrand's theorem [9, 3]: a formula is valid if and only if there is a finite expansion (of existential quantifiers to disjunctions and universal quantifiers to conjunctions of instances) which is a propositional tautology. Such Herbrand expansions can be transformed to and obtained from cut-free sequent calculus proofs in a quite straightforward way.

It is non-trivial to formally extend this separation to proofs with cuts. An approach which has been successful in this respect is the use of tree grammars, introduced in [10] for proofs with $\Pi_{1}$-cuts and extended to $\Pi_{2}$-cuts in [1, 2]. In this setting, a proof in sequent calculus induces a tree grammar which bears all instances of the end-sequent as well as the instantiation structure of the cuts without direct reference to the cut formulæ themselves: one obtains a Herbrand expansion by computing the language of the grammar.

In addition to the proof-theoretic interest behind an abstract representation of proofs with cut, proof grammars provide a number of applications. Motivated by the aim to structure and compress automatically generated proofs, an algorithm for cut-introduction based on proof grammars has been developed in [13, 12]. This method has been implemented and empirically evaluated with good results in [11]. An extension of these techniques to the case of proofs with $\Pi_{1}$-induction has led to a new technique for inductive theorem proving [6] which is currently being implemented. A final application of proof grammars is in the area of proof complexity, where lower bounds on the length of proofs with cuts (which are notoriously difficult to control) are obtained by transferring lower bounds on the size of the corresponding grammar [5, 4].

There are other formalisms which allow Herbrand expansions to be computed in a way that abstracts from the propositional structure. The historically first such formalism is Hilbert's $\varepsilon$-calculus [15]. In [7] Gerhardy and Kohlenbach adapt Shoenfield's variant of Gödel's Dialectica interpretation to a system of pure predicate logic. Recent work, related to proof nets, is that of Heijltjes [8] and McKinley [16], and a similar approach, in the formalism of expansion trees [17], can be found in [14]. What sets proof grammars apart from these formalisms is that they not only compute Herbrand expansions but provide a (well-understood) abstract description of its structure which is crucial for the applications mentioned above.

U. Kohlenbach, S. van Bakel, S. Berardi (Eds.): CL\&C'16 EPTCS 213, 2016, pp. 1-10 doi 10.4204/EPTCS.213.1 (c) B. Afshari, S. Hetzl \& G.E. Leigh

This work is licensed under the Creative Commons Attribution License. 
In the present paper we provide an intermediate formalism between proof grammars and functional interpretations with the aim of studying the relationship between the two approaches. This intermediate formalism is presented as a grammar but instead of capturing the instantiation structure directly, it is given by a brief line-by-line definition on the proof, as functional interpretations usually are. The necessity for computing more than one witness (reflected by the case distinction constants in the GerhardyKohlenbach version of the Dialectica interpretation [7]) is reflected by non-deterministic production rules in the grammar.

The main result we prove in this paper is stated below. Note that in the presence of Skolemisation it suffices to consider proofs with $\Sigma_{1}$ end sequents.

Theorem 1.1. Let $\pi$ be a proof of $\exists \boldsymbol{v} F$ with $F$ quantifier-free in which cut-formula are prenex $\Pi_{2}$ or $\Sigma_{2}$. There exists an acyclic context-free grammar $\mathscr{G}$ such that $\bigvee_{\boldsymbol{t} \in L(\mathscr{G})} F(\boldsymbol{t})$ is valid. Moreover, $L(\mathscr{G})$ contains the Herbrand set extracted from any cut-free proof that can be obtained from $\pi$ via a sequence of cut reductions (see Figure 2) that always reduces to the weak (quantifier) side of a cut before the strong side.

More generally, $L(\mathscr{G})$ covers the Herbrand set of any cut-free proof obtained from $\pi$ by a sequence of reductions fulfilling the following two restrictions.

1. A contraction on a universally quantified $\Pi_{2}$ formula is reduced only when no other reduction rule is applicable (to this cut);

2. If two cuts are permuted in the form

$$
\text { cut } \frac{A, B, \Gamma \quad \bar{A}, \Delta}{\operatorname{cut} \frac{B, \Gamma, \Delta}{\Gamma, \Delta, \Lambda} \bar{B}, \Lambda} \quad \rightsquigarrow \quad \text { cut } \frac{A, B, \Gamma \quad \bar{B}, \Lambda}{\operatorname{A,\Gamma }, \Lambda} \bar{A}, \Delta
$$

then one of $A$ and $B$ is not $\Pi_{2}$.

\section{The system LK}

Traditionally LK is represented in two-sided sequent calculus. For notational simplicity however, we work in one-sided sequent calculus (Tait-style) with explicit weakening (w), contraction (c) and permutation (p) rules. Axioms and rules are laid out in Figure 1 and the cut reduction steps are presented in Figure 2. We generally leave applications of the permutation rule implicit: its only role is to facilitate defining the grammar in the next section.

\begin{tabular}{|c|c|c|c|}
\hline Axioms: & $A, \bar{A}$ & for $A$ an atomic $\mathrm{f}$ & \\
\hline \multirow{6}{*}{ Inference rules: } & $A, B, \Gamma$ & $A, \Gamma \quad B, \Delta$ & \\
\hline & $A \vee B, \Gamma$ & $\overline{A \wedge B, \Gamma, \Delta}$ & \\
\hline & $A(v / \alpha), \Gamma$ & $A(v / t), \Gamma$ & $A, \Gamma \quad \bar{A}, \Delta$ \\
\hline & $\forall v A, \Gamma$ & $\exists v A, \Gamma$ & $\Gamma, \Delta$ \\
\hline & $\Gamma$ & $A, A, \Gamma$ & $\Gamma, B, A, \Delta$ \\
\hline & $\overline{A, \Gamma}$ & $\overline{A, \Gamma}$ & $\overline{\Gamma, A, B, \Delta}$ \\
\hline
\end{tabular}

Figure 1: Axioms and rules of LK. The usual eigenvariable conditions for quantifier introduction apply. 


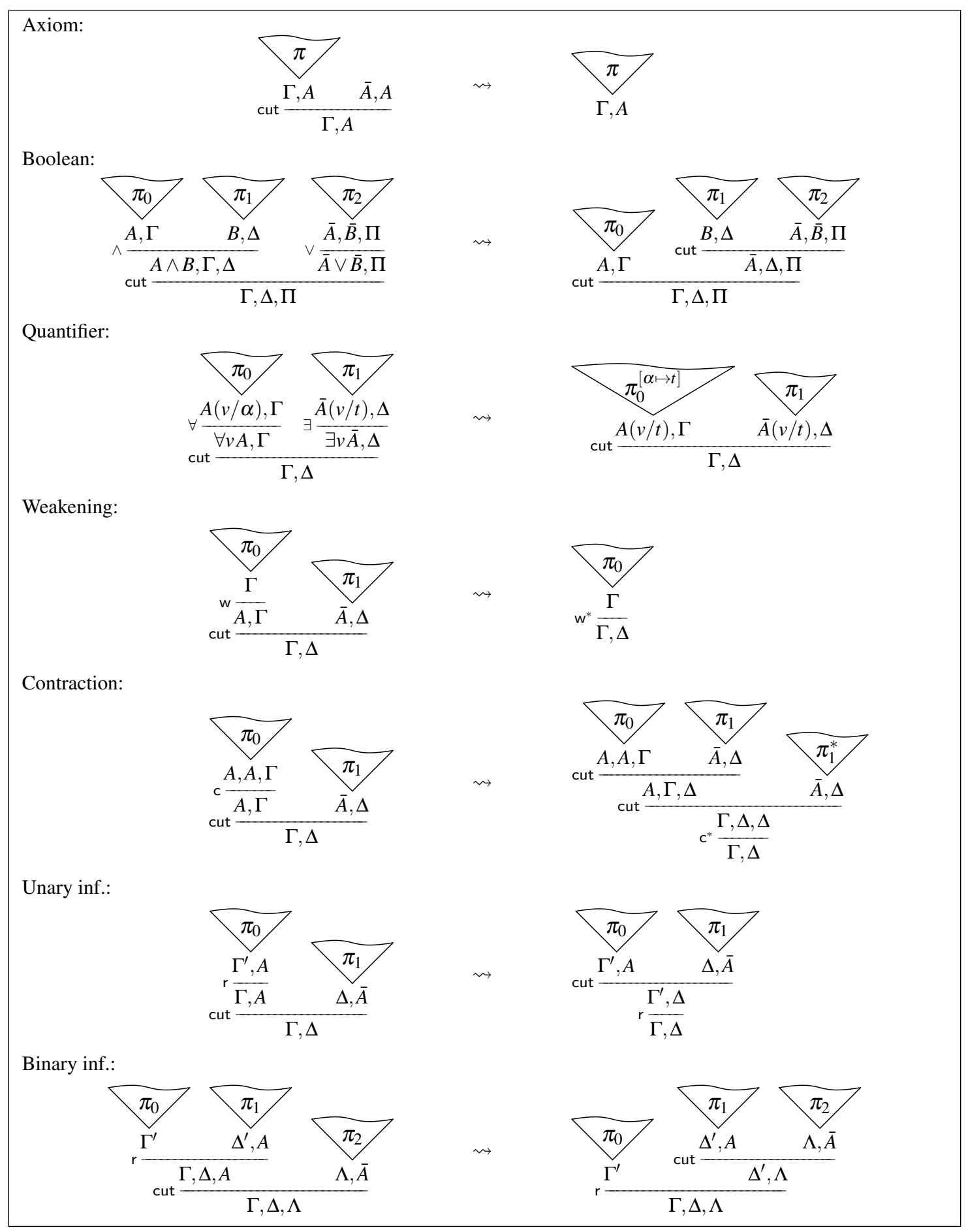

Figure 2: One-step cut reduction and permutation rules. In the final two reductions, $r$ denotes respectively an arbitrary unary and binary rule. 
We use $\alpha, \beta$, etc. for free and $v, w$, etc. for bound variables. Upper-case Roman letters, $A$, $B$, etc. denote formulæ and upper-case Greek letters $\Gamma, \Delta$, etc. range over sequents, namely finite sequences of formulæ. We write $\bar{A}$ to denote the dual of the formula $A$ obtained by de Morgan laws. A proof is a finite binary tree labelled by sequents obtained from the axioms and rules of the calculus with the restriction that cuts apply to prenex $\Pi_{2} / \Sigma_{2}$ formulæ only. Without loss of generality, we assume all proofs are regular, namely strong quantifier inferences are associated unique eigenvariables. This is particularly relevant in the case of contraction reduction where a sub-proof is duplicated and eigenvariables renamed (expressed by annotating the proof in question by an asterisk) to maintain regularity. The length of a sequent $\Gamma$ is denoted $|\Gamma|$ and we write $\pi \vdash \Gamma$ to express that $\pi$ is a proof with end sequent $\Gamma$.

\section{Proof grammars}

To an LK-proof $\pi \vdash \Gamma$ we associate a typed non-deterministic tree grammar $\mathscr{G}_{\pi}$ (equivalently, an order- 2 recursion scheme) with production rules that abstract the computation of Herbrand sets achieved through Gentzen-style cut-elimination.

Informally, $\mathscr{G}_{\pi}$ comprises rewrite rules for symbols $\sigma_{\pi^{\prime}}^{i}$ where $\pi^{\prime} \vdash \Gamma^{\prime}$ is a sub-proof of $\pi$ and $0 \leq$ $i<\left|\Gamma^{\prime}\right|$. The non-terminal $\sigma_{\pi^{\prime}}^{i}$ is of function type (of order $\leq 2$ ) with arity $\left|\Gamma^{\prime}\right|$ returning a sequence of closed terms as witnesses for the weak quantifiers in the $i$-th formula in $\Gamma^{\prime}$. The $j$-th argument of $\sigma_{\pi^{\prime}}^{i}$ is interpreted as input for the strong quantifiers in the $j$-th formula in $\Gamma^{\prime}$ and is either a finite sequence (of determined length) or a function from first-order objects to sequences thereof, the case depending on the quantifier complexity of the corresponding formula. The type of the arguments to $\sigma_{\pi^{\prime}}^{i}$ is independent of $i$.

As an example, consider a derivation $\pi \vdash A_{0}, A_{1}$ where $A_{0}$ and $A_{1}$ are prenex $\Sigma_{2} \cup \Pi_{2}$ formulæ with $m_{0}$ and $m_{1}$ existential quantifiers respectively. The grammar contains two non-terminals associated to $\pi$, $\sigma_{\pi}^{0}$ and $\sigma_{\pi}^{1}$, of type

$$
\sigma_{\pi}^{0}: \tau_{0} \rightarrow \tau_{1} \rightarrow(\underbrace{o \times \cdots \times o}_{m_{0}})
$$

$$
\sigma_{\pi}^{1}: \tau_{0} \rightarrow \tau_{1} \rightarrow(\underbrace{o \times \cdots \times o}_{m_{1}})
$$

where $o$ denotes the type of first-order terms and $\tau_{i}$ is a type depending on the number of universal quantifiers in $A_{i}$. Given terms $T_{0}: \tau_{0}$ and $T_{1}: \tau_{1}$ the grammar rewrites the term $\sigma_{\pi}^{i} T_{0} T_{1}$ to a sequence of first-order terms $\left\langle t_{1}, \ldots, t_{m_{i}}\right\rangle$ of length $m_{i}$ to be interpreted as witnesses to the extensional quantifiers in $A_{i}$. The role of $T_{0}$ and $T_{1}$ is to provide input for the strong quantifiers (specifically, their corresponding eigenvariables) on which witnesses to the existential quantifiers may depend. For instance, if $A_{i}=\forall v \exists w_{0} \exists w_{1} B_{i}$ and $B_{i}$ is quantifier-free for each $i$, then $\tau_{0}=\tau_{1}=o, m_{0}=m_{1}=2$ and $\sigma_{\pi}^{i} T_{0} T_{1}$ rewrites to pairs of the form $\langle r, s\rangle\left[\alpha \mapsto T_{0}\right]\left[\beta \mapsto T_{1}\right]$ where $r$ and $s$ are first-order terms and $\alpha$ and $\beta$ are the eigenvariables for the strong quantifier in $A_{0}$ and $A_{1}$ respectively. Higher-type terms arise in the case of sequents with $\Sigma_{2}$ formulæ. Suppose $A_{0}=\exists v \forall w B_{0}$ where $B_{0}$ is quantifier-free and $A_{1}$ is as above. In this case $T_{0}$ has type $\tau_{0}=(o \rightarrow o)$ and is utilised in generating input for the universal quantifier in $A_{0}$ modulo witnesses for the existential quantifier. If the final inference in $\pi$ derives the sequent $A_{0}, A_{1}$ from $\pi_{0} \vdash \forall w B_{0}(v / r), A_{1}$ then since $B_{0}$ is quantifier-free, the two non-terminals associated to $\pi_{0}$ are

$$
\sigma_{\pi_{0}}^{0}: o \rightarrow o \rightarrow o \quad \sigma_{\pi_{0}}^{1}: o \rightarrow o \rightarrow(o \times o) .
$$

The production rules corresponding to this inference are

$$
\sigma_{\pi}^{0} T_{0} T_{1} \rightarrow\langle r\rangle \quad \sigma_{\pi}^{1} T_{0} T_{1} \rightarrow \sigma_{\pi_{0}}^{1}\left(T_{0} r\right) T_{1} .
$$


The left-hand rule returns the term $r$ as the (single) witness to the existential quantifier in $A_{0}$ whereas the right-hand rule records the fact that in a witness to the existential quantifier in $A_{1}$, any occurrence of the eigenvariable for the universal in $A_{0}$ will be substituted for $T_{0} r$. In general, $T_{0}$ will itself contain non-terminals for other parts of the (wider) proof and will have been introduced through the nesting of non-terminals that occurs when passing through a cut rule (see Figure 3).

In the following, fix an LK-proof $\pi \vdash \Gamma$ in which all formulæ are prenex $\Pi_{2} / \Sigma_{2}$.

\subsection{Terms and types}

We expand first-order terms by a form of explicit substitution, resulting in structured (first-order) terms: every first-order term is a structured term, and if $s$ and $t$ are structured terms and $\alpha$ is a free-variable symbol then the expression $s[\alpha \mapsto t]$ is a structured term.

Let $o$ denote the type of structured first-order terms and $\varepsilon$ the unit type with a single element \langle\rangle$: \varepsilon$. $o$ and $\varepsilon$ are called ground-types and their elements ground-terms. We consider the explicit substitution constructors above as term building operations on both $o$ and $\varepsilon$. A type hierarchy is formed by closing the ground-types under the usual pair-types and function-types: given $u, x: \rho$ and $u^{\prime}: \rho^{\prime}$ we have $u \star u^{\prime}: \rho \times \rho^{\prime}$ and $\lambda x u^{\prime}: \rho \rightarrow \rho^{\prime}$. To avoid unnecessary parenthesis the three binary (infix) operations are assumed to associate to the right. We define $o^{0}=\varepsilon$ and $o^{k+1}=o \times o^{k}$. An element of a type of the form $o^{k}$ is called a sequence-term. Given a (possibly empty) sequence $\left(u_{i}: o\right)_{i<k}$ of terms of type $o$, we write $\left\langle u_{0}, \ldots, u_{k-1}\right\rangle$ to abbreviate the sequence-term $u_{0} \star \cdots \star u_{k-1} \star\langle\rangle$ of type $o^{k}$.

\begin{tabular}{|c|c|}
\hline Rule of inference & Corresponding production rule(s) \\
\hline$\pi \vdash \bar{A}, A$ & $\sigma_{\pi}^{i} z_{0} z_{1} \rightarrow z_{1-i}$ \\
\hline$\pi_{0} \vdash A(v / \alpha), \Gamma$ & \\
\hline$\forall-\forall v A, \Gamma$ & $\sigma_{\pi}^{l}\left(z_{0} \star z_{1}\right) \boldsymbol{y} \rightarrow\left(\sigma_{\pi_{0}}^{l} z_{1} \boldsymbol{y}\right)\left\lfloor\alpha \mapsto z_{0}\right]$ \\
\hline$\pi_{0} \vdash A(v / r), \Gamma$ & $\sigma^{i}, \quad \int r \star\left(\sigma_{\pi_{0}}^{0}(z \cdot r) \boldsymbol{y}\right), \quad$ if $i=0$ \\
\hline$\pi \vdash \exists v A, \Gamma$ & $\sigma_{\pi} \varepsilon \boldsymbol{y} \rightarrow \begin{cases}\sigma_{\pi_{0}}^{i}(z \cdot r) \boldsymbol{y}, & \text { otherwise. }\end{cases}$ \\
\hline$\pi_{0} \vdash A, \Gamma \quad \pi_{1} \vdash \bar{A}, \Delta$ & $\boldsymbol{x} \boldsymbol{y} \rightarrow\left\{\sigma_{\pi_{0}}^{i+1}\left(\left(\sigma_{\pi_{1}}^{0} \circ_{\bar{A}} \sigma_{\pi_{0}}^{0}\right) \boldsymbol{y} \boldsymbol{x}\right) \boldsymbol{x}, \quad i<|\Gamma|\right.$ \\
\hline$\pi \vdash \Gamma, \Delta$ & 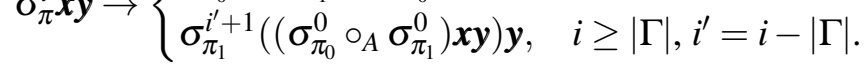 \\
\hline$\pi_{0} \vdash A, A, \Gamma$ & $\int \sigma_{\pi_{0}}^{0} z z \boldsymbol{y} \mid \sigma_{\pi_{0}}^{1} z z \boldsymbol{y}, \quad$ if $i=0$ \\
\hline$\pi \vdash A, \Gamma$ & $\sigma_{\pi_{0}}^{i+1} z z \boldsymbol{y}, \quad 0<i<|\Gamma|$. \\
\hline${ }_{w} \frac{\pi_{0} \vdash \Gamma}{2}$ & $\sigma_{-i}^{i} z \boldsymbol{y} \rightarrow\left\{\mathrm{c}_{A}, \quad\right.$ for $i=0$ \\
\hline$\pi \vdash A, \Gamma$ & $\sigma_{\pi} \boldsymbol{y} \rightarrow\left\{\sigma_{\pi_{0}}^{i-1} \boldsymbol{y}, \quad\right.$ otherwise. \\
\hline$\pi_{0} \vdash \Gamma, B, A, \Delta$ & $\begin{cases}\sigma_{\pi_{0}}^{i+1} x_{z_{1}} z_{0} \boldsymbol{y}, & \text { if } i=|\Gamma|,\end{cases}$ \\
\hline $\bar{\pi} \overline{\pi \vdash \Gamma, A, B, \Delta}$ & $\boldsymbol{x} z_{0} z_{1} \boldsymbol{y} \rightarrow \begin{cases}\sigma_{\pi_{0}}^{l-1} \boldsymbol{x} z_{1} z_{0} \boldsymbol{y}, & \text { if } i=|\Gamma|+1 \\
\sigma_{\pi_{0}}^{i} \boldsymbol{x}_{z_{1}} z_{0} \boldsymbol{y}, & \text { otherwise. }\end{cases}$ \\
\hline
\end{tabular}

Figure 3: Production rules: $\boldsymbol{x}$ and $\boldsymbol{y}$ denote sequences of distinct variables of length $|\Gamma|$ and $|\Delta|$ respectively; the contraction rule is the only inference introducing non-determinism; $\mathrm{c}_{k}, \circ_{F}$ and $z \cdot r$ are abbreviations for terms described in Section 3.2 . 
Let $\Gamma=\left\{A_{0}, \ldots, A_{n}\right\}$. The type of $\sigma_{\pi}^{i}$ is given by

$$
\sigma_{\pi}^{i}: \tau_{A_{0}}^{*} \rightarrow \cdots \rightarrow \tau_{A_{n}}^{*} \rightarrow \tau_{A_{i}}
$$

where $\tau_{F}$ and $\tau_{F}^{*}$ are determined by the complexity of $F$ :

- for $F=\forall v_{1} \cdots \forall v_{m} \exists w_{1} \cdots \exists w_{n} G$ with $G$ quantifier-free,

$$
\tau_{F}=o^{n}=o^{m}
$$

- for $F=\exists v_{1} \cdots \exists v_{m} \forall w_{1} \cdots \forall w_{n} G$ with $n>0$ and $G$ quantifier-free,

$$
\tau_{F}=o^{m}=\underbrace{o \rightarrow \cdots \rightarrow o}_{m} \rightarrow o^{n} .
$$

The $\operatorname{order}$ of a type $\rho$ (and term of type $\rho$ ), $\operatorname{ord}(\rho)$, is defined as usual: $\operatorname{ord}(\varepsilon)=\operatorname{ord}(o)=0$, $\operatorname{ord}\left(\rho \times \rho^{\prime}\right)=\max \left\{\operatorname{ord}(\rho), \operatorname{ord}\left(\rho^{\prime}\right)\right\}$ and $\operatorname{ord}\left(\rho \rightarrow \rho^{\prime}\right)=\max \left\{\operatorname{ord}(\rho)+1, \operatorname{ord}\left(\rho^{\prime}\right)\right\}$. Thus for a proof $\pi \vdash \Gamma$ where $\Gamma$ is a set of prenex $\Pi_{2}$ and $\Sigma_{2}$ formulæ, and for $i<|\Gamma|$, the order of $\sigma_{\pi}^{i}$ is no greater than 2 .

In the sequel we avoid explicit mention of types when they can be inferred from context.

\subsection{Production rules}

Let $\Sigma$ be a finite set of (typed) variable symbols. A structured $\lambda$-term over $\Sigma$ is a well-typed term constructed from ground-terms, variables and non-terminals via the term-forming operations described above in which any freely occurring variable is an element of $\Sigma$.

The production rules for non-terminals are determined by the final rule applied to the index proof and are presented in Figure 3. Each production rule has the form $u \rightarrow u^{\prime}$ where the two terms are of the same ground-type and $u^{\prime}$ is a structured $\lambda$-term over the free variables in $u$. With the exception of the $\forall$ production rules (which are discussed below) $u$ has the form $\sigma_{\pi}^{i} x_{0} \cdots x_{n}$ which is often condensed to $\sigma_{\pi}^{i} \boldsymbol{x}$. Note that the contraction rule is the only inference rule that introduces non-determinism.

The presentation of the production rules includes the following abbreviations. The symbol $c_{A}$ appearing in the production rule for weakening $(\mathrm{w})$ denotes the sequence-term $\langle\mathrm{c}, \ldots, \mathrm{c}\rangle: o^{k}$ where $\mathrm{c}: o$ is some fixed constant symbol and $k$ is the number of existential quantifiers in $A$.

The binary operation · appearing in the rule for existential quantifiers $(\exists)$ extends term application to cases in which the first argument has type $\varepsilon$ in a trivial way:

$$
z \cdot x= \begin{cases}z, & \text { if } z: \varepsilon \\ z x, & \text { otherwise. }\end{cases}
$$

Its role is to compensate for the case that $A$ is a $\Sigma_{1}$ formula whereby $\tau_{\exists v A}^{*}=\tau_{A}^{*}=\varepsilon$; otherwise $\tau_{\exists v A}^{*}=$ $o \rightarrow \tau_{A}^{*}$ and so $z \cdot r: \tau_{A}^{*}$ as required.

In the production rules for cut (cut), the operation $\circ_{F}$ abbreviates combining two non-terminals depending on the quantifier complexity of $F$. Let $\pi_{0} \vdash \Gamma$ and $\pi_{1} \vdash \Delta$ be LK-proofs, and $i<|\Gamma|, j<|\Delta|$. For non-terminals $\sigma_{\pi_{0}}^{0}$ and $\sigma_{\pi_{1}}^{0}$ and variable sequences $\boldsymbol{x}$ and $\boldsymbol{y}$ of length $|\Gamma|-1$ and $|\Delta|-1$ respectively, we define

$$
\left(\sigma_{\pi_{0}}^{0} \circ_{F} \sigma_{\pi_{1}}^{0}\right) \boldsymbol{x} \boldsymbol{y}= \begin{cases}\langle\rangle, & \text { if } F \text { is quantifier-free } \\ \lambda z_{0} \cdots \lambda z_{m} . \sigma_{\pi_{0}}^{0}\left\langle z_{0}, \ldots, z_{m}\right\rangle \boldsymbol{x}, & \text { if } F=\forall v_{0} \cdots \forall v_{m} G \text { and } G \in \Sigma_{1}, \\ \sigma_{\pi_{0}}^{0}\left(\lambda z_{0} \cdots \lambda z_{m} . \sigma_{\pi_{1}}^{0}\left\langle z_{0}, \ldots, z_{m}\right\rangle \boldsymbol{y}\right) \boldsymbol{x}, & \text { if } F=\exists v_{0} \cdots \exists v_{m} G \text { and } G \in \Pi_{1}\end{cases}
$$


Observe that according to the typing introduced earlier, the production rules listed in Figure 3 are welltyped (by definition, the axiom case applies only when $A$ is quantifier-free whence all relevant types are identical).

Before we proceed with the definition of language it is important to address the universal introduction rule $\forall$. In its stated form, $\mathscr{G}_{\pi}$ is context-sensitive as the reduction depends on the form of at least one argument. A context-free grammar can be obtained by formulating the production rules using projection functions for pair-types:

$$
\sigma_{\pi}^{n} z \boldsymbol{y} \rightarrow\left(\sigma_{\pi_{0}}^{n}\left(p_{1} z\right) \boldsymbol{y}\right)\left[\alpha \mapsto p_{0} z\right]
$$

Doing so, however, will in general expand the language of the grammar: if $T$ is a term that nondeterministically rewrites to $r_{0} \star T_{0}$ and $r_{1} \star T_{1}$ (and the four terms are pairwise distinct) then substituting $T$ for $z$ in (1) yields four combinations of terms, compared with just two available from the $\forall$ production rule. Nevertheless, this increase will be always finite.

\subsection{Language}

A derivation in the grammar is a sequence of structured $\lambda$-terms containing non-terminals obtained by applying a sequence of grammar production rules and $\beta$-reductions. For a well-typed term $u$ (possibly containing non-terminals), $L(u)$ denotes the set of terms derivable from $u$ to which no further rules may be applied. We write $u \sim u^{\prime}$ if $L(u)=L\left(u^{\prime}\right)$.

Given a sequence-term $T=\left\langle t_{1}, \ldots, t_{k}\right\rangle: o^{k}$ of structured first-order terms, let $T^{*}$ denote the result of evaluating all explicit substitutions occurring in $T$, forming a sequence of first-order terms. Fix a sequent $\Gamma=\exists \boldsymbol{v}_{0} A_{0}, \ldots, \exists \boldsymbol{v}_{k} A_{k}$ of prenex $\Sigma_{1}$ formulæ wherein for each $i \leq k, A_{i}$ is quantifier-free and $\exists \boldsymbol{v}_{i}$ abbreviates a block of existential quantifiers of length $a_{i}$. Let $\pi \vdash \Gamma$ be an LK-proof with cuts of complexity at most prenex $\Pi_{2} / \Sigma_{2}$. The language of $\pi$, denoted $L(\pi)$, is the set of pairs $\left(i, T^{*}\right)$ such that $i \leq k$ and $T: o^{a_{i}}$ is a structured sequence-term free of non-terminal symbols derivable from the term $\sigma_{\pi}^{i}\langle\rangle \cdots\langle\rangle$. The next lemma demonstrates that the choice of starting symbol is canonical.

Lemma 3.1. If $\pi \vdash A, \Gamma$ and $A$ is prenex $\Sigma_{1}$ then for all terms $u_{0}, \ldots, u_{|\Gamma|}$ of the appropriate type we have $\sigma_{\pi}^{i} u_{0} \cdots u_{|\Gamma|} \sim \sigma_{\pi}^{i}\langle\rangle u_{1} \cdots u_{|\Gamma|}$.

Since the production rules are naturally acyclic (rewriting a non-terminal $\sigma_{\pi}^{i}$ introduces only nonterminals indexed by strict sub-proofs of $\pi$ ), we deduce

Lemma 3.2. For any regular proof $\pi, L(\pi)$ is finite.

As a consequence of Lemma 3.2 the language of a proof $\pi$ can be viewed as inducing an expansion of its end-sequent, obtained by replacing each formula $\exists \boldsymbol{v}_{i} A_{i}$ by the corresponding disjunction $\bigvee\left\{A_{i}(\boldsymbol{t}) \mid\right.$ $(i, \boldsymbol{t}) \in L(\pi)\}$.

Theorem 3.3. If $\pi \vdash \Gamma$ is an $L K$-proof of a prenex $\Sigma_{1}$ sequent in which all cuts are prenex $\Pi_{2}$ and $\Sigma_{2}$ formula then the expansion of $\Gamma$ induced by $L(\pi)$ is a tautology.

The proof of this theorem (and the more general statement in Theorem 1.1) is covered in Section 4 below by establishing that the language of a proof is preserved through most cut reduction steps. In the (base) case that all cuts in $\pi$ are on quantifier-free formulæ, we observe that the grammar rules merely associate to each weak quantifier in the end-sequent the witnesses as they appear in $\pi$. 


\section{Language preservation}

Let $\pi \rightsquigarrow \pi^{\prime}$ express that $\pi^{\prime}$ is obtained from $\pi$ by the application of a reduction rule in Figure 2 to a subproof of $\pi$. In the present section we determine for which reduction steps $\pi \rightsquigarrow \pi^{\prime}$ we have: (i) language inclusion: $L(\pi) \supseteq L\left(\pi^{\prime}\right)$; and (ii) language equality: $L(\pi)=L\left(\pi^{\prime}\right)$. Language inclusion will suffice to derive the main theorem; equality allows a more fine-grained study of the Herbrand content of proofs as if $\pi_{0}$ and $\pi_{1}$ are proofs that can be connected by a sequence of forward and backward language-preserving reduction steps then $L\left(\pi_{0}\right)=L\left(\pi_{1}\right)$.

The structure of our proof grammars is such that to deduce inclusion or equality it suffices to analyse the reduction steps locally:

\subsection{Cut permutation}

We begin by considering the instances of the binary inference reduction that permute two cuts. Suppose $\pi \rightsquigarrow \pi^{\prime}$ are the two proofs
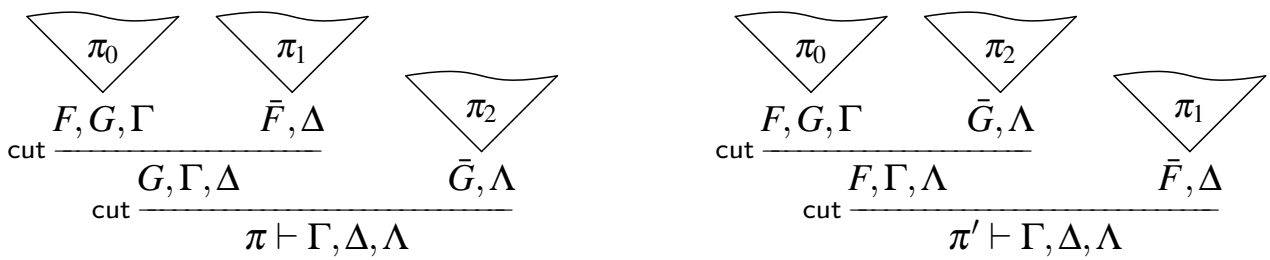

Lemma 4.1. For $\pi$ and $\pi^{\prime}$ in (2), if either $F$ or $G$ is $\Sigma_{2}$ then $L(\pi)=L\left(\pi^{\prime}\right)$.

Lemma 4.1 covers all cases of permuting two cuts that suffice for establishing Theorem 1.1. In the case both $F$ and $G$ are (genuine) $\Pi_{2}$ formulæ, the language of the grammars need not be preserved:

Lemma 4.2. There are instantiations of $\pi$ and $\pi^{\prime}$ in (2) such that $L(\pi)$ and $L\left(\pi^{\prime}\right)$ are incomparable.

\subsection{Contraction reduction}

Consider the proofs
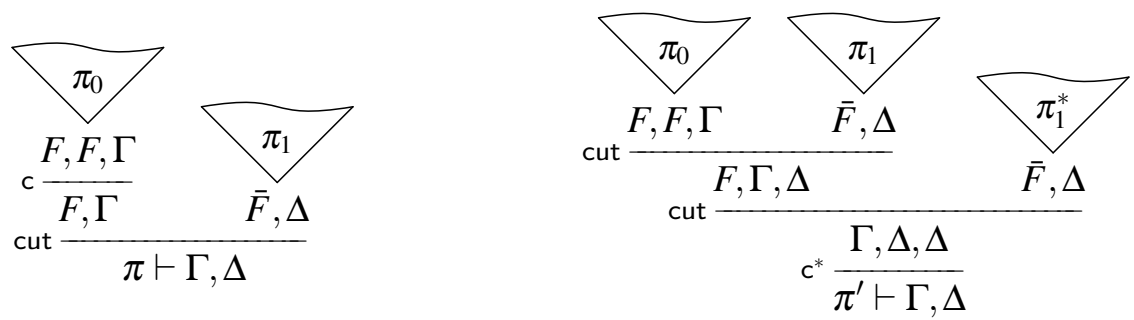

Lemma 4.3. For $\pi$ and $\pi^{\prime}$ in (3), if $F$ is $\Sigma_{2}$ then $L(\pi)=L\left(\pi^{\prime}\right)$.

As in the previous case, language inclusion does not hold in general when reducing a contraction. Specifically, if (i) $F$ is a genuine $\Pi_{2}$ formula i.e. $F=\forall v_{0} \cdots \forall v_{k} \exists w G$ for some $\Sigma_{1}$ formula $G$, and (ii) there are contractions on $\bar{F}$ in the subproof $\pi_{1}$ then the languages $L(\pi)$ and $L\left(\pi^{\prime}\right)$ can be incomparable. We do, however, have

Lemma 4.4. For $\pi$ and $\pi^{\prime}$ in $(3)$, if $F$ is $\Pi_{2}$ and there are no contractions on $\bar{F}$ in the sub-proof $\pi_{1}$ then $L\left(\pi^{\prime}\right) \subseteq L(\pi)$. 


\subsection{Quantifier reduction}

Lemma 4.5. For $\pi$ and $\pi^{\prime}$ in (4) we have $L(\pi)=L\left(\pi^{\prime}\right)$.

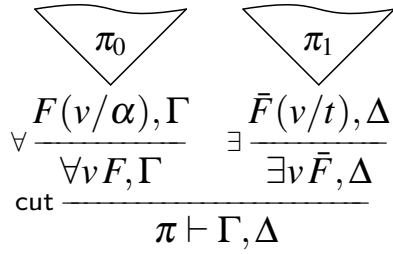

\subsection{Quantifier permutation}

Consider permuting a universal quantifier with a cut:

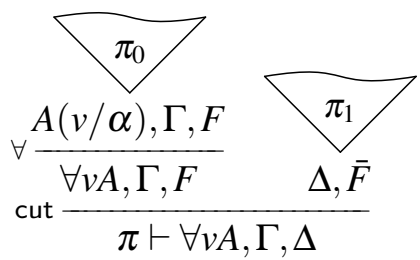

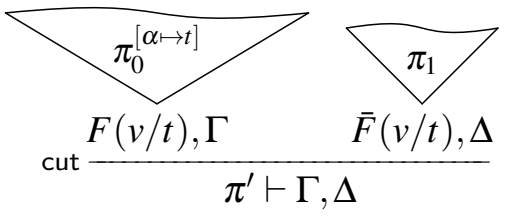

Lemma 4.6. For $\pi$ and $\pi^{\prime}$ in (5) we have $L\left(\pi^{\prime}\right) \subseteq L(\pi)$.

\subsection{Remaining reductions}

The remaining rules are straightforward to analyse and all induce language equality except for weakening reduction for which we have language inclusion.

\section{Conclusion}

To each proof in first order logic with prenex $\Pi_{2} / \Sigma_{2}$ cuts we associate a formal grammar abstracting the semantic aspect of cut elimination and classify the cut reduction and permutation rules according to whether or not the language of the grammar is preserved under these rules. The ultimate goal of the study is to extend this classification to arbitrary classes of cut-formulæ.

The grammars utilised in this paper have a number of advantages over previous language-theoretic approaches for proofs with $\Pi_{2} / \Sigma_{2}$ cuts [1, 2]. We can now deal with non-simple proofs (i.e. proofs admitting contractions on universal formulæ) as well as blocks of like quantifiers. Furthermore, unlike the grammars devised in [1, 2], derivations are not restricted by equality constraints (or rigidity requirements). As a result, preservation of language over the cut reduction steps reduces more or less to mere computation. Also notable is the fact that the production rules of the grammar given here are both acyclic and unidirectional relative to the associated proof: each production rule rewrites a non-terminal in favour of non-terminals labelled by strict sub-proofs. Finally, the new grammars make the appearance of non-confluence in cut-elimination more transparent.

Acknowledgements The authors' research was supported by the Wiener Wissenschafts-, Forschungsund Technologiefonds (WWTF), project no. VRG12-04. The authors wish to thank the anonymous referees for their helpful comments and suggestions. 


\section{References}

[1] Bahareh Afshari, Stefan Hetzl \& Graham E. Leigh (2015): Herbrand disjunctions, cut elimination and context-free tree grammars. In Thorsten Altenkirch, editor: 13th International Conference on Typed Lambda Calculi and Applications (TLCA 2015), Leibniz International Proceedings in Informatics (LIPIcs) 38, Schloss Dagstuhl-Leibniz-Zentrum fuer Informatik, Dagstuhl, Germany, pp. 1-16, doi $10.4230 /$ LIPIcs.TLCA.2015.1

[2] Bahareh Afshari, Stefan Hetzl \& Graham E. Leigh (2016): Herbrand confluence for first-order proofs with $\Pi_{2}$-cuts. In Dieter Probst \& Peter Schuster, editors: Concepts of Proof in Mathematics, Philosophy, and Computer Science, Ontos Mathematical Logic, De Gruyter, Berlin, Boston. isbn: 978-1-5015-0262-0. Preprint avaiable at http://dmg.tuwien.ac.at/afshari/publications.html.

[3] Samuel R. Buss (1995): On Herbrand's theorem. In: Logic and Computational Complexity, Lecture Notes in Computer Science 960, Springer, pp. 195-209, doi:10.1007/3-540-60178-3_85.

[4] Sebastian Eberhard \& Stefan Hetzl: On the compressibility of finite languages and formal proofs. In preparation, preprint available at http://www.logic.at/people/hetzl/research/

[5] Sebastian Eberhard \& Stefan Hetzl (2015): Compressibility of finite languages by grammars. In Jeffrey Shallit \& Alexander Okhotin, editors: Descriptional Complexity of Formal Systems (DCFS) 2015, Lecture Notes in Computer Science 9118, Springer, pp. 93-104, doi 10.1007/978-3-319-19225-3_8.

[6] Sebastian Eberhard \& Stefan Hetzl (2015): Inductive theorem proving based on tree grammars. Annals of Pure and Applied Logic 166(6), pp. 665 - 700, doi 10.1016/j.apal.2015.01.002.

[7] Philipp Gerhardy \& Ulrich Kohlenbach (2005): Extracting Herbrand disjunctions by functional interpretation. Archive for Mathematical Logic 44, pp. 633-644, doi 10.1007/s00153-005-0275-1.

[8] Willem Heijltjes (2010): Classical proof forestry. Annals of Pure and Applied Logic 161(11), pp. 1346-1366, doi $10.1016 /$ j.apal.2010.04.006.

[9] Jacques Herbrand (1930): Recherches sur la théorie de la démonstration. Ph.D. thesis, Université de Paris.

[10] Stefan Hetzl (2012): Applying tree languages in proof theory. In Adrian-Horia Dediu \& Carlos Martín-Vide, editors: Language and Automata Theory and Applications (LATA) 2012, Lecture Notes in Computer Science 7183, Springer, pp. 301-312, doi 10.1007/978-3-642-28332-1_26.

[11] Stefan Hetzl, Alexander Leitsch, Giselle Reis, Janos Tapolczai \& Daniel Weller (2014): Introducing quantified cuts in logic with equality. In Stéphane Demri, Deepak Kapur \& Christoph Weidenbach, editors: Automated Reasoning - 7th International Joint Conference, IJCAR, Lecture Notes in Computer Science 8562, Springer, pp. 240-254, doi:10.1007/978-3-319-08587-6_17.

[12] Stefan Hetzl, Alexander Leitsch, Giselle Reis \& Daniel Weller (2014): Algorithmic introduction of quantified cuts. Theoretical Computer Science 549, pp. 1-16, doi:10.1016/j.tcs.2014.05.018.

[13] Stefan Hetzl, Alexander Leitsch \& Daniel Weller (2012): Towards algorithmic cut-introduction. In Andrei Bjørner, Nikolaj \& Voronkov, editor: Logic for Programming, Artificial Intelligence and Reasoning (LPAR18), Lecture Notes in Computer Science 7180, Springer, pp. 228-242, doi:10.1007/978-3-642-28717-6_19.

[14] Stefan Hetzl \& Daniel Weller (2013): Expansion trees with cut. Preprint available at http://arxiv.org/ abs/1308.0428

[15] David Hilbert \& Paul Bernays (1939): Grundlagen der Mathematik II. Springer.

[16] Richard McKinley (2013): Proof nets for Herbrand's theorem. ACM Transactions on Computational Logic 14(1), pp. 5:1-5:31, doi:10.1145/2422085.2422090.

[17] Dale Miller (1987): A compact representation of proofs. Studia Logica 46(4), pp. 347-370, doi:10.1007/BF00370646. 